\title{
von Willebrand's Disease with Spontaneous Platelet Aggregation Induced by an Abnormal Plasma von Willebrand Factor
}

\author{
Harvey R. Gralnick, Sybil B. Williams, Laurie P. McKeown, and Margaret E. Rick \\ Hematology Service, Clinical Pathology Department, Clinical Center, National Institutes of Health, Bethesda, Maryland 20205
}

Pascal Maisonneuve, Christine Jenneau, and Yvette Sultan

Hopital Cochin, Paris, France

\begin{abstract}
We have investigated and characterized the abnormalities in four unrelated patients with von Willebrand's disease ( $v W d)$ who have (a) enhanced ristocetin-induced platelet aggregation (RIPA) at low ristocetin concentrations, $(b)$ absence of the largest plasma von Willebrand factor (vWf) multimers, and (c) thrombocytopenia. The platelet-rich plasma of these patients aggregates spontaneously without the addition of any agonists. When isolated normal platelets are resuspended in patient plasma spontaneous aggregation occurs; however, the patients' plasmas did not induce platelet aggregation of normal washed formalinized platelets. When the patients' platelets are suspended in normal plasma, spontaneous aggregation is not observed. The spontaneous platelet aggregation (SPA) is associated with dense granule secretion as measured by ATP release and alpha granule release as measured by $\beta$-thromboglobulin and platelet factor 4 release. The SPA is totally inhibited by $5 \mathrm{mM}$ EDTA, prostaglandin $\mathrm{I}_{2}$, and dibutryl cyclic AMP, while it is only partially inhibited by 1 mM EDTA, acetylsalicylic acid, or apyrase. A monoclonal antibody directed against glycoprotein Ib (GPIb) and/or a monoclonal antibody against the glycoprotein IIb/IIIa (GPIIb/ IIIa) complex totally inhibits the SPA.

The vWf was isolated from the plasma of one of these patients. The purified vWf induced platelet aggregation of normal platelets resuspended in either normal or severe vWd plasma, but the $\mathrm{vWf}$ did not induce platelet aggregation of normal platelets resuspended in afibrinognemic plasma. Sialic acid and galactose quantification of the patient's $\mathrm{vWf}$ revealed approximately a 50\% reduction compared with normal vWf. These studies indicate that a form of vWd exists, which is characterized by SPA that is induced by the abnormal plasma vWf. The SPA is dependent on the presence of plasma fibrinogen, and the availability of the GPIb and the GPIIb/IIIa complex. In this variant form of vWd the abnormal vWf causes enhanced RIPA, SPA, and thrombocytopenia.
\end{abstract}

\section{Introduction}

Variant forms of von Willebrand's disease $(\mathrm{vWd})^{1}$ with enhanced ristocetin-induced platelet aggregation (RIPA) have recently been separated into two major groups, classified as type IIb and platelet

Address correspondence to Dr. Gralnick, Hematology Service, CPD, Clinical Center, National Institutes of Health, Building 10, Room 2C390, Bethesda, MD 20205. 1985.

Received for publication 13 March 1985 and in revised form 3 July

The Journal of Clinical Investigation, Inc.

Volume 76, October 1985, 1522-1529 or pseudo-vWd (1-3). The multimeric structure of Factor VIII/ von Willebrand factor (F.VIII/vWf) present in the plasma of these two groups of patients is indistinguishable. The mechanism of the enhanced RIPA in the type IIb vWd is related to enhanced binding of the plasma von Willebrand factor (vWf) to patient or normal platelets in the presence of low concentrations of ristocetin. In pseudo-vWd or platelet-type vWd, the plasma vWf defect is secondary to a platelet abnormality that results in the adsorption of the largest multimeric forms of plasma $\mathrm{vWf}$ to platelets independent of ristocetin. Since the plasmas of these patients lack the largest multimers, platelet aggregation ensues only when normal vWf in the form of cryoprecipitate or purified protein (possessing the largest multimers) is infused in vivo or added in vitro to their platelet-rich plasma (PRP) or when these multimeric forms are released in vivo after I-desamino-8-D-arginine vasopressin (DDAVP) infusion $(2,4-6)$.

We report four individuals from four different families who represent a form of $\mathbf{v W d}$ different from those previously described with increased RIPA. All four are characterized by enhanced RIPA at low doses of ristocetin, abnormal multimeric structure of their plasma vWf, spontaneous platelet aggregation (SPA) of their PRP, and thrombocytopenia. Their plasmas induce platelet aggregation of normal platelets when the latter are resuspended in the patients' plasmas. Monoclonal antibodies to either the vWf binding site on glycoprotein Ib (GPIb) or the fibrinogen binding site on the glycoprotein IIb/IIIa (GPIIb/IIIa) complex inhibit the SPA.

The purified plasma vWf isolated from one patient causes SPA when added to normal PRP. The aggregation is dependent on the presence of plasma fibrinogen. The patient's purified $\mathrm{vWf}$ contains $45 \%$ of the sialic acid and $60 \%$ of the galactose content of normal vWf. We and others have shown that normal or partially desialyated vWf induces platelet aggregation that is dependent on the presence of plasma fibrinogen and is totally inhibited by monoclonal antibodies against GPIb or GPIIb/IIIa (7-11). We believe that the triad of enhanced RIPA, SPA, and thrombocytopenia is caused by circulating Asialo vWf (AS-vWf) in this particular variant form of $\mathrm{vWd}$.

\section{Methods}

Blood was obtained via a 19-gauge needle using a two-syringe technique. Plasma collection, preparation of PRP, separation of platelets from whole

1. Abbreviations used in this paper: AS, Asialo; $\beta \mathrm{TG}, \beta$-thromboglobulin; db-cAMP, dibutyl cyclic AMP; DDAVP, I-desamino-8-D-arginine vasopressin; F.VIII/vWf, Factor VIII/von Willebrand factor; GPIb, glycoprotein Ib; GPIIb/IIIa, glycoprotein IIb/IIIa; PF4, platelet factor 4; $\mathrm{PGI}_{2}$, prostaglandin $\mathrm{I}_{2} ; \mathrm{PRP}$, platelet-rich plasma; RIPA, ristocetin-induced platelet aggregation; SPA, spontaneous platelet aggregation; VIII: C, Factor VIII coagulant activity; VIII R:Ag, Factor VIII-related antigen; vWd, von Willebrand's disease; vWf, von Willebrand's factor. 
blood, and purification of normal and patient F.VIII/vWf protein were performed as previously described $(12,13)$. Cryoprecipitate was prepared from $240 \mathrm{ml}$ of fresh frozen plasma. $10 \mathrm{U}$ of cryoprecipitate were used to purify normal and/or patient vWf. The purpose of this study was explained to the patients; they gave informed consent for venipuncture and blood collection. Factor VIII coagulant activity (VIII:C), Factor VIIIrelated antigen (VIII R:Ag), and vWf activities were measured as previously described (13). One unit is defined as that found in $1 \mathrm{ml}$ of pooled normal plasma.

Patient platelets were analyzed for VIII R:Ag and vWf activities after isolation from whole blood. Platelets were isolated from whole blood anticoagulated with $10.9 \mathrm{mM}$ sodium citrate, $1 \mathrm{mM}$ EDTA on a discontinuous arabinogalactan (Stractan; St. Regis Co., Takoma, WA) gradient ( $3 \mathrm{ml} \mathrm{20 \%}$ and $5 \mathrm{ml} \mathrm{10 \%} \mathrm{Stractan)} \mathrm{as} \mathrm{previously} \mathrm{described} \mathrm{(14).}$ The platelets were washed free of Stractan with $0.01 \mathrm{M}$ Tris, $0.15 \mathrm{M}$ $\mathrm{NaCl}, 0.003 \mathrm{M}$ EDTA, $0.5 \%$ bovine serum albumin, $\mathrm{pH}$ 6.8. After centrifugation at $1,500 \mathrm{~g}$ for $10 \mathrm{~min}$ the platelet pellet was resuspended in $0.01 \mathrm{M}$ tris, $0.15 \mathrm{M} \mathrm{NaCl}, 3 \%$ bovine serum albumin, $\mathrm{pH} 7.35$, incubated at $37^{\circ} \mathrm{C}$ for $15 \mathrm{~min}$, and then counted. The platelet concentration was adjusted to $10^{6} / \mu \mathrm{l}$ by the addition of the $\mathrm{pH} 7.35$ buffer, and the platelets were lysed by the addition of $1 / 40$ volume of $20 \% \mathrm{wt} / \mathrm{wt}$ Triton X-100. The platelets were frozen at $-70^{\circ} \mathrm{C}$, and before analysis they were thawed and spun at $10,000 \mathrm{~g}$ for $30 \mathrm{~min}$ at $4^{\circ} \mathrm{C}$. The supernatant was removed and used in the assays for VIII R:Ag and vWf.

Platelet aggregation. Aggregation studies were performed in either a Chronolog or Payton Dual Channel aggregometer. Identical teflon-coated stirring bars were used with the patients' PRP and normal PRP. PRP was prepared from whole blood anticoagulated with $3.2 \%$ sodium citrate ( $10.9 \mathrm{mM}$, final concentration) by centrifugation at $750 \mathrm{~g}$ for $3 \mathrm{~min}$ at room temperature; the platelet count was adjusted to $200,000 / \mu 1$ by the addition of the appropriate platelet-poor plasma and then was placed in an air-tight polypropylene container. RIPA of normal PRP and platelet PRP was performed as follows: $0.4 \mathrm{ml}$ of PRP (platelet count, 200,000/ $\mu l$ except for one patient with a PRP that contained $160,000 / \mu \mathrm{l}$ ) was placed in an aggregometer cuvette, the stir bar was added, and a 30-s base line was established. Ristocetin was added at concentrations ranging from $0.25-2.0 \mathrm{mg} / \mathrm{ml}$. For each set of experiments, the patient PRP and normal PRP were prepared at the same time and were run alternately immediately after the preparation of the PRP.

SPA was examined on PRP that was prepared and tested as above except that no agonists were added. The blood for PRP preparation was collected in three different anticoagulants: (a) the original sodium citrate, $3.2 \%, 10.9 \mathrm{mM}$ final concentration; (b) a mixture of sodium citrate, 10.9 mM with $1 \mathrm{mM}$ EDTA; and $(c)$ same as $(b)$ except that the EDTA was $5 \mathrm{mM}$ final concentration. The PRP was placed in the aggregometer and the aggregation tracing was followed for at least $15 \mathrm{~min}$.

For mixing studies normal or patient platelets were isolated from whole blood on an arabinogalactan gradient as above with the following modifications. The blood was anticoagulated with $10.9 \mathrm{mM}$ sodium citrate and the platelets were adjusted to $200,000 / \mu$ l by resuspension in the Hepes buffer or normal or patients' plasma and tested for SPA. In some studies the platelets were formalin-fixed, resuspended in normal or patients' plasma at a final concentration of $200,000 / \mu \mathrm{l}$, and tested for SPA in the presence or absence of $2 \mathrm{mM} \mathrm{CaCl}$ or $2 \mathrm{mM} \mathrm{MgCl}_{2}$. The patients' plasmas included the four patients described in this report, one patient with severe vWd (plasma VIII:C, <3\%; VIII R:Ag, <5\%; vWf activity, $<3 \%$; platelet vWf activity and antigen, $<3 \%$ ) previously described (10), and two patients with afibrinogenemia. Platelets from the patient with severe $\mathrm{vWd}$ were separated from citrate-anticoagulated whole blood as described above and were resuspended in patient or normal plasma and then tested for SPA.

Release of ATP and platelet factor 4 (PF4) and $\beta$-thromboglobulin ( $\beta T G)$ were measured in some experiments. ATP release was quantitated by the luciferase-luciferin reagent (Sigma Chemical Co., St. Louis, MO) in the Chrono-Lumi aggregometer as per the manufacturer's instructions. PF4 and $\beta$ TG release were measured by radioimmunoassay using commercially available kits (Abbott Laboratories, Irving, TX; and Amersham Corp., Arlington Heights, IL). To quantitate the PF4 and $\beta$ TG release at specific time points, an equal volume of $2 \%$ formalin in 0.05 Tris, $0.10 \mathrm{NaCl}$, pH 7.35 was added to the PRP $(0.4 \mathrm{ml}, 200,000 / \mu \mathrm{l})$. The tube was inverted twice for rapid mixing and then placed at $4^{\circ} \mathrm{C}$ for 20 min. The formalin-fixed PRP was centrifuged in an Eppendorf Microfuge at $12,000 \mathrm{~g}$ for $4 \mathrm{~min}$. An aliquot of the supernatant plasma was removed from the top of the tube and assayed. Controls included normal and patient plasma separated from the PRP, which was fixed before being placed in the aggregometer, and the plasma was separated from PRP and fixed after sitting (at $37^{\circ} \mathrm{C}$ ) for $20 \mathrm{~min}$ without stirring.

In some studies $50-100 \mu$ of either fresh normal platelet-poor plasma, normal freshly prepared human cryoprecipitate $(1.5-3.7 \mathrm{vWf} \mathrm{U} / \mathrm{ml}$, final concentration), or freshly prepared normal purified vWf protein (50-75 $\mathrm{vWf} \mathrm{U} / \mathrm{ml}$, final concentration) was added to the normal PRP or patient PRP. The aggregation tracings were followed for $15 \mathrm{~min}$ and compared with the same PRP without the addition of exogenous vWf.

Inhibitors of platelet aggregation were tested with either patients' PRP or normal platelets suspended in patients' plasma to examine their effect on the SPA. EDTA was used in two of the original anticoagulants in which the blood was collected at 1 and $5 \mathrm{mM}$ final concentration (see above). Dibutryl cyclic AMP (db-cAMP) 4.5-20 mM (Sigma Chemical Co.); apyrase, 20-200 $\mu \mathrm{g} / \mathrm{ml}$ (Sigma Chemical Co.); and prostaglandin $\mathrm{I}_{2}$ ( $\mathrm{PGI}_{2}$ ), 4-48 $\mu \mathrm{M}$ (Sigma Chemical Co.) and Hiruidin, 20-40 U/ml (Sigma Chemical Co.) were added to PRP 3 min before adding the stirring bar. The $\mathrm{PGI}_{2}$ was diluted in Tris buffer, $\mathrm{pH}$ 8.6. Acetyl salicylic acid $(0.45-0.90 \mathrm{mg} / \mathrm{ml}$; Aldrich Chemical Co., Milwaukee, WI) was diluted in Tris, $0.05 \mathrm{M} ; \mathrm{NaCl}, 0.10 ; \mathrm{pH} 7.8$, or $0.3 \mathrm{M}$ sodium acetate. It was added to the PRP $30 \mathrm{~min}$ before the stirring bar. Comparison of the initial slope and total spontaneous aggregation of the PRP without the inhibitors was compared with those in the presence of the inhibitors.

Two well-characterized monoclonal antibodies, 6D1 directed against glycoprotein Ib, and 10E5 directed against the GPIIb/IIIa complex, were added to the PRP in the form of purified IgG or as the $\mathrm{Fab}_{2}$ fragment $(10,15,16)$. The monoclonal antibodies were added to the PRP 3 min before adding the stirring bar. The amount of purified monoclonal antibody added varied from 0.02 to $13.0 \mu \mathrm{g} / \mathrm{ml}$. A monoclonal antibody specific for the T4 subset of human lymphocytes was used as a control monoclonal antibody. (The T4 antibody was purchased from Coulter Electronics, Inc., Hialeah, FL).

Human vWf was purified from $10 \mathrm{U}$ of cryoprecipitate by gel chromatography on Sepharose 4B as previously described (13).

Total sialic acid content of purified vWf was analyzed after hydrolysis at $80^{\circ} \mathrm{C}$ for $60 \mathrm{~min}$ in $0.1 \mathrm{~N} \mathrm{H}_{2} \mathrm{SO}_{4}$ according to the method of Warren (17). $\mathrm{N}$-acetyl neuraminic acid V (Sigma Chemical Co.) was used as a standard.

Terminal and penultimate galactose were measured after the treatment of the intact or Asialo vWf with $\beta$-galactosidase purified from Streptococcus pneumoniae. The enzyme, a gift of Dr. Gilbert Ashwell, was free of proteolytic activity and was stabilized with $3 \mathrm{mg} / \mathrm{ml}$ of bovine serum albumin. The vWf protein was incubated before and after neuraminidase treatment with $\beta$-galactosidase $0.02-0.05 \mathrm{U} / \mathrm{mg}$ protein for 8 h. The free galactose was measured by the method of Finch et al. (18). Total galactose was measured after hydrolysis of the $v W f$ in $2 \mathrm{~N} \mathrm{HCl}$ for $1 \mathrm{~h}$ at $100^{\circ} \mathrm{C}$ as previously described (19). The sample was then assayed for galactose by the method of Finch (18).

The multimeric structure of the normal plasma and patients' plasma and the platelet vWf were studied by glyoxyl agarose electrophoresis modified from the technique originally described by Hoyer and Shainoff (20). The major modifications were a gel thickness of $0.5 \mathrm{~mm}$ and a final sample size of $10 \mu \mathrm{l}$.

Reduced and nonreduced purified normal $\mathrm{vWf}$ and patient $\mathrm{vWf}$ were electrophoresed on $5 \%$ polyacrylamide gels and on $0.5 \%$ agarose- $2 \%$ acrylamide gels in the presence of SDS as previously described (13).

\section{Results}

Each of the four patients has been studied at least four times. On each examination the bleeding time has been long, the VIII: 


\begin{tabular}{|c|c|c|c|c|c|c|c|c|c|c|c|}
\hline \multirow[b]{3}{*}{ Patient } & \multirow{3}{*}{$\begin{array}{l}\text { Bleeding } \\
\text { time }\end{array}$} & \multirow[b]{3}{*}{ VIII:C } & \multirow[b]{3}{*}{ VIII R:Ag } & \multirow[b]{3}{*}{$\mathbf{v W f}$} & \multirow{3}{*}{$\begin{array}{l}\text { Platelet } \\
\text { count }\end{array}$} & \multirow{2}{*}{\multicolumn{4}{|c|}{ RIPA* ristocetin }} & \multicolumn{2}{|l|}{$\mathrm{SPA}^{*}$} \\
\hline & & & & & & & & & & \multirow{2}{*}{$\begin{array}{l}\text { Initial } \\
\text { velocity }\end{array}$} & \multirow[b]{2}{*}{ Lag } \\
\hline & & & & & & 0.25 & 0.50 & 1.0 & 2.0 & & \\
\hline & $\min$ & $U / m l$ & $U / m l$ & $U / m l$ & $\times 10^{3} / \mu l$ & $\mathrm{mg} / \mathrm{ml}$ & $m g / m l$ & $m g / m l$ & $m g / m l$ & $\mathrm{~mm} / \mathrm{min}$ & $\min$ \\
\hline 1 & 15 & 0.34 & 0.53 & 0.35 & $82-117 \ddagger$ & 0 & 12 & 27 & 58 & $6-11$ & $0.5-4.5$ \\
\hline 2 & $>20$ & 0.26 & 0.75 & 0.45 & $28-159$ & 11 & 21 & 35 & 60 & $5-18$ & $0.5-7.0$ \\
\hline 3 & $>30$ & 0.38 & 0.50 & 0.18 & $33-69$ & 0 & 18 & 23 & 60 & $9-24$ & $0.2-5.0$ \\
\hline 4 & 17 & 0.39 & 0.60 & 0.30 & $61-131$ & 10 & 28 & 60 & 55 & $7-40$ & $0.5-2.0$ \\
\hline Normal & $<9$ & $0.52-1.58 \S$ & $0.55-1.68 \S$ & $0.59-1.43 \S$ & $143-365 \S$ & 0 & 4 & 10 & 62 & N.D. & - \\
\hline
\end{tabular}

N.D., not detectable. The coagulation and platelet data represent the mean value of at least three determinations. ${ }^{*}$ RIPA, ristocetin-induced platelet aggregation of PRP. The values given for RIPA and SPA are the initial slope in $\mathrm{mm} / \mathrm{min}$. The normal values are the means of the results on the PRP of six normals for RIPA and 14 normals for SPA. Two of the normals had $1 \mathrm{~mm} / \mathrm{min}$ SPA. $¥$ Range of platelet counts observed over a 3-yr period. $\S$ Normal range derived from mean $\pm 2 \mathrm{SD}$.

$\mathrm{C}$ and vWf activity has been reduced, and the VIII R:Ag has been slightly decreased or normal (Table I). The platelet count has varied in all four patients from moderately to mildly decreased; however, two platelet counts out of a total of 24 were normal. The SPA has been a consistent finding in these patients over a period of $3 \mathrm{yr}$. We have seen variation in the initial slope, the length of the lag time, and the total amount of aggregation; however, each patient has been tested at least four separate times and has always shown SPA (Table I). The addition of normal $\mathrm{vWf}$ (in normal plasma, normal cryoprecipitate, or purified vWf) to the patients' PRP did not augment any aspect of the SPA. The multimeric structure of the patients' plasma vWf is variable, but all lacked the largest multimers (Fig. 1). Slight variations were seen in the amount of intermediate multimers. In three of the four patients the platelet vWf contained a full complement of multimers; however, in one patient (patient 2), the largest multimers were lacking and in patient three there was an increase in the concentration of all multimers (Fig. 2 and Table II). In these experiments, the platelet $\mathrm{vWf}$ from $10^{9}$ platelets was isolated and an aliquot was electrophoresed.

The studies to identify whether the patients' platelets or plasma were responsible for the SPA were performed by resuspending normal platelets in patient plasma and resuspending patient platelets in normal plasma. The patients' platelets did not spontaneously aggregate when they were suspended in either normal plasma or buffer, while both normal and patient platelets spontaneously aggregated when suspended in the patients' PPP (Fig. 3). The results were similar when the plasma and platelets of all four patients were studied. The lag time and slope of aggregation varied when normal platelets were resuspended in patients' plasma; however, the plasma of all four patients induced SPA of normal platelets. The platelets of the four patients did not aggregate when resuspended in normal plasma. When normal or patient platelets were resuspended in patient plasma, the lag period and initial velocity of platelet aggregation were similar to the SPA observed with the patients' own PRP. The platelets from a patient with severe $\mathrm{vWd}$ also aggregated spontaneously when resuspended in patients' plasma. In contrast, formalinized platelets did not undergo SPA when they were resuspended in the patient plasma. The addition of $\mathrm{CaCl}_{2}$ or $\mathrm{MgCl}_{2}$ (final concentration, $2 \mathrm{mM}$ ) did not alter the results.

The SPA of the patients' PRP or the SPA of normal platelets resuspended in patient plasma was associated with the release of alpha granule constituents as measured by the release of $\beta$ TG and PF4 and dense granules as measured by the release of ATP (Fig. 4). The PRP of patients 3 and 4 were studied and both showed similar results. The release of ATP in the patient PRP or patient plasma and normal platelets varied from $0.11-0.45$

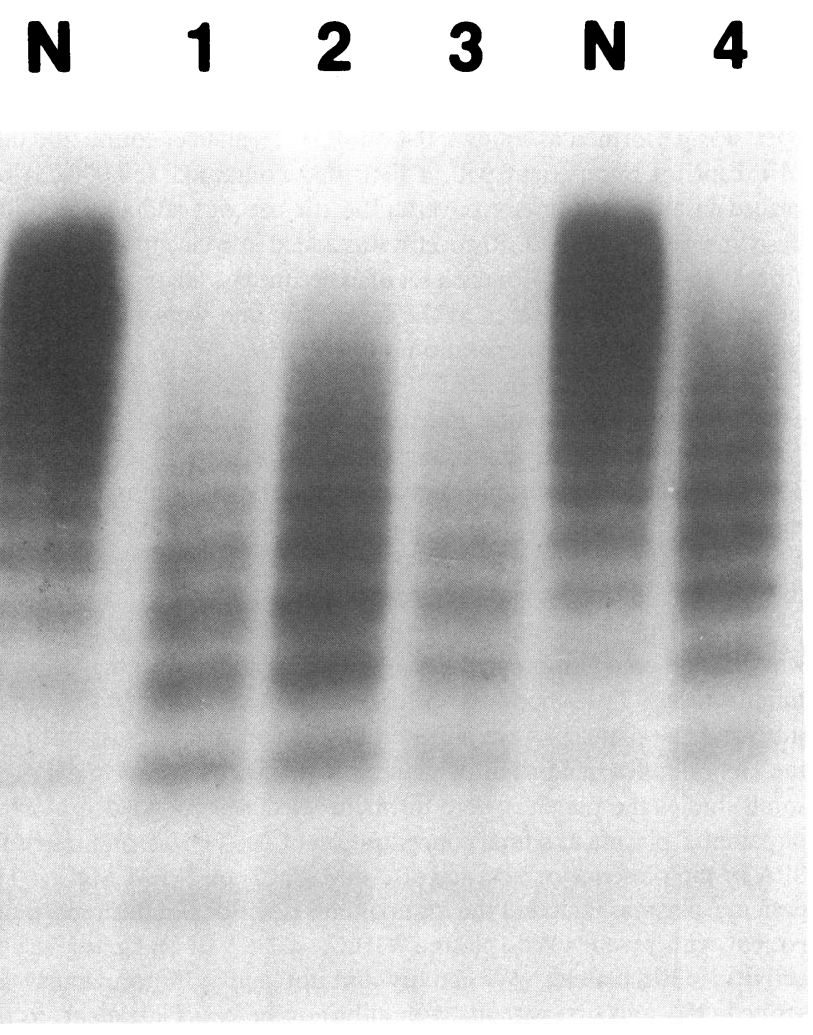

Figure 1. Agarose gel electrophoresis of plasma vWf. Patient plasma samples were analyzed on glyoxyl agarose gel electrophoresis (gel concentration, $1.25 \%, 0.5 \mathrm{~mm}$ thick). $10 \mu \mathrm{l}$ of each sample was applied to the gel. No corrections were made for antigen plasma content. $\mathrm{N}$ represents normal plasma, and the numbers 1, 2, 3, and 4 represent the same patients as described in Table $I$. All four patients lacked the largest vWf multimers, and there is a variable decrease in the intermediate sized multimers in all four patient plasmas. Anode is at the bottom of the figure. 


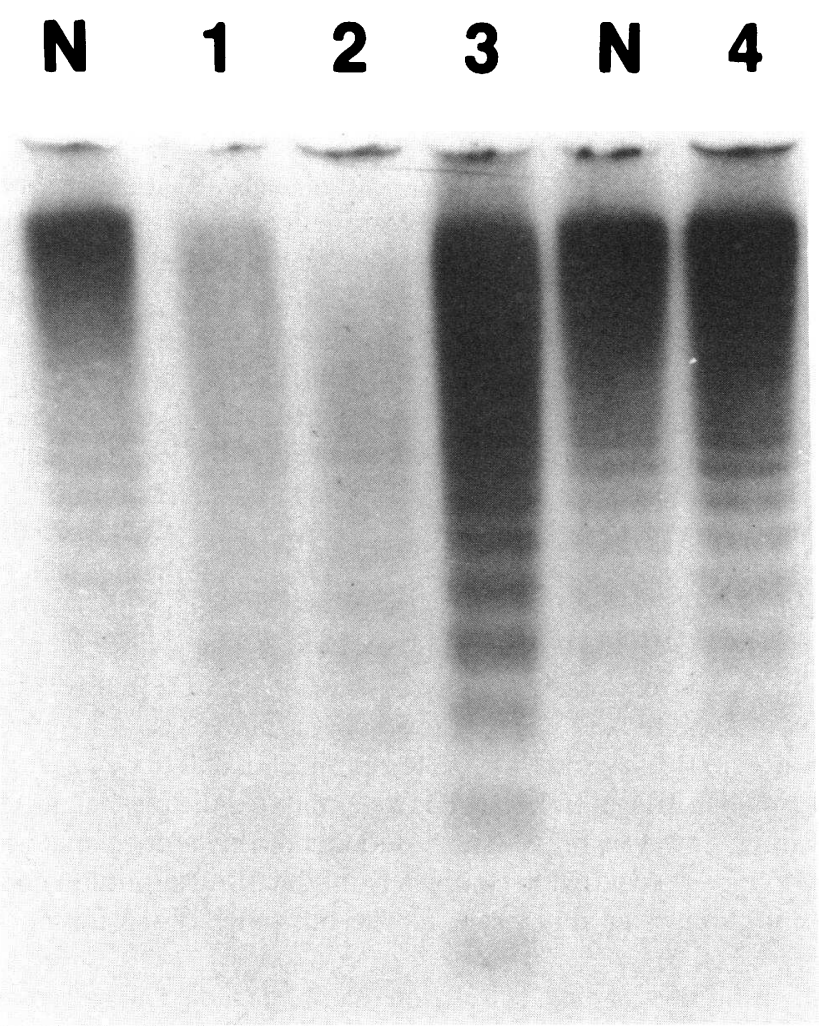

Figure 2. Agarose gel electrophoresis of platelet vWf multimers. Glyoxyl agarose gel electrophoresis was performed at a gel concentration of $1.25 \%$, thickness $0.5 \mathrm{~mm}$. $10 \mu \mathrm{l}$ of sample was applied to the gel. All samples were tested at $10^{9}$ platelets $/ \mathrm{ml}$. $\mathrm{N}$ represents two different normal platelets and the numbers 1, 2, 3, and 4 represent the same patients as described in Tables I and II. Patients 1, 3, and 4 have a full complement of the platelet vWf multimers, while patient 2 has an absence of the largest vWf multimers. The antigen units for each patient's sample electrophoresed and shown in the figure were: patient $1,0.30 \mathrm{U} / 10^{9}$ platelets (range, $0.39-0.58 \mathrm{U} / 10^{9}$ platelets, $n=3$ ); patient $2,0.20 \mathrm{U} / 10^{9}$ platelets (range, $0.18-0.30 \mathrm{U} / 10^{9}$ platelets, $n=3$ ); patient $3,0.87 \mathrm{U} / 10^{9}$ platelets (range, $0.63-0.87 \mathrm{U} / 10^{9}$ platelets, $n$ $=4$ ); and patient $4,0.55 \mathrm{U} / 10^{9}$ platelets (range, 0.44-0.72 U/109 platelets, $n=4)$. The normal platelet vWf on the far left contained $0.38 \mathrm{U} / 10^{9}$ platelets and the normal platelet $\mathrm{vWf}$ in the next-to-last lane on the right contained $0.51 \mathrm{U} / 10^{9}$ platelet (normal range, $0.26-$ $0.58 \mathrm{U} / 10^{9}$ platelet). Anode is at the bottom of the figure.

Table II. Plasma and Platelet VIII R:Ag and vWf Activities

\begin{tabular}{llllll}
\hline & \multicolumn{3}{l}{ Plasma } & & Platelet \\
\cline { 2 - 3 } \cline { 5 - 6 } Patient & Antigen & $\mathrm{vWf}$ & & Antigen & $\mathrm{vWf}^{*}$ \\
\hline & & & & $\begin{array}{l}U / 10^{9} \\
\text { platelets }\end{array}$ & $\begin{array}{l}U / 10^{9} \\
\text { platelets }\end{array}$ \\
& & $U / m l$ & & \\
1 & 0.53 & 0.35 & & 0.46 & 0.75 \\
2 & 0.75 & 0.45 & & 0.23 & 0.28 \\
3 & 0.50 & 0.18 & & 0.73 & 0.60 \\
4 & 0.60 & 0.30 & 0.58 & 1.42 \\
Normal mean $\neq$ & 0.96 & 1.06 & 0.44 & 0.91 \\
& $(0.55-1.68)$ & $(0.59-1.43)$ & $(0.26-0.58)$ & $(0.67-1.27)$
\end{tabular}

* $1 \mathrm{ml}$ contained $10^{9}$ platelets.

$¥$ Mean and range derived from the values obtained from 12 normal donors. Range is the mean \pm 2 SD.

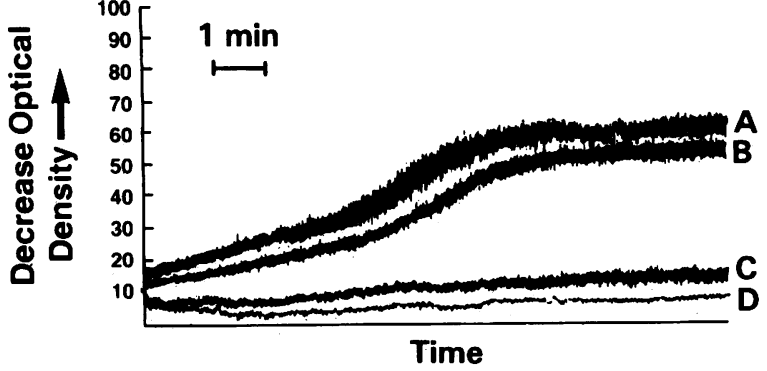

Figure 3. Platelet aggregation studies. PRP $(200,000$ platelets/ $\mu$ l) 0.4 $\mathrm{ml}$ was placed in the aggregometer at $37^{\circ} \mathrm{C}$ and stirred at $900 \mathrm{rpm}$. Tracing A shows the SPA in the patient's PRP (patient 2, Table I). Tracing $B$ demonstrates the results when normal platelets isolated from whole blood are resuspended in patient plasma. Tracing $\mathrm{C}$ depicts normal platelets isolated from whole blood resuspended in normal plasma. Tracing $D$ depicts the patient's platelets isolated from whole blood resuspended in normal plasma. Only those samples that contain patient plasma showed SPA. The patient's platelets did not support SPA.

$\mu \mathrm{M}$. In contrast, normal PRP or patient platelet resuspended in normal plasma did not release ATP (Fig. 4).

The plasma-induced SPA was not dependent on the presence of platelet vWf, since the platelets from a patient with severe $\mathrm{vWd}$ with no detectable platelet vWf aggregated to the same degree as did normal platelets when they were resuspended in the patients' plasma. To further clarify the plasma proteins involved in the SPA, the vWf was purified from one of these patients (patient 4, Table I). The patient's purified vWf protein

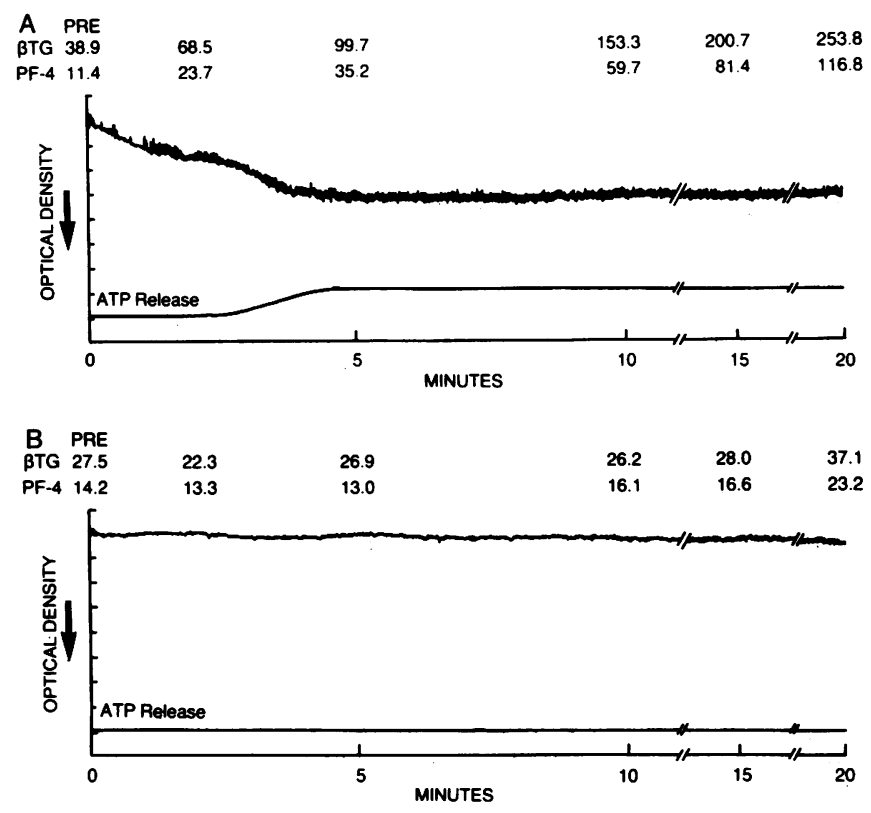

Figure 4. Platelet aggregation of patient PRP and release of dense granule and alpha granule constituents. $(A) 0.4 \mathrm{ml}$ of PRP (patient 3 , Table I) $(200,000 / \mu \mathrm{l})$ was placed in an aggregometer at $37^{\circ} \mathrm{C}$ at 900 rpm. At specified times, the PRP was fixed and prepared for $\beta$-thromboglobulin and PF4 determinations (see Methods). ATP release was monitored by leuciferase-leuciferin reaction. Release of alpha and dense granule constituents occurred after the initiation of aggregation. ATP release was $0.34 \mu \mathrm{M}$ (range, 0.11-0.45 $\mu \mathrm{M}$ ). (B) Normal PRP. Note that the aggregation curve(s) are oriented opposite to those in the other figures. 
(300 $\mu \mathrm{g} / \mathrm{ml}$ ) contained $<0.5 \mu \mathrm{g} / \mathrm{ml}$ fibrinogen as determined by tanned erythrocyte hemagglutination inhibition. Analysis of the patient vWf on $5 \%$ polyacrylamide gel electrophoresis revealed a band at the top of the gel and after reduction, a single band was present with a relative molecular weight $\left(M_{\mathrm{r}}\right)$ of 220,000 , similar to the band seen with normal vWf $\left(M_{\mathrm{r}}\right.$ of 220,000$)$ (Fig. $5)$. According to our calculation, the difference in the molecular weight of the vWf due to the sialic and galactose deficiency was $\sim 4,000$ less than normal. This value was just at the limit of sensitivity of the $5 \%$ polyacrylamide gel electrophoresis system.

When the purified $v W f$ was added to normal PRP at a final concentration of $5.5-22.0 \mu \mathrm{g} / \mathrm{ml}$, SPA occurred. At $2.75 \mu \mathrm{g} / \mathrm{ml}$ aggregation was seen in one of three experiments. When the patients' purified vWf (lowest active amount, $5.5 \mu \mathrm{g} / \mathrm{ml}$ ) was added to isolated normal platelets resuspended in either normal plasma or in the plasma from a patient with severe vWd, they underwent SPA. However, when normal platelets were resuspended in afibrinogenemic plasma, the purified $\mathrm{vWf}$ from the vWd patients did not induce SPA even at $33 \mu \mathrm{g} / \mathrm{ml}$.

Sialic acid content of the patient's vWf revealed a decrease
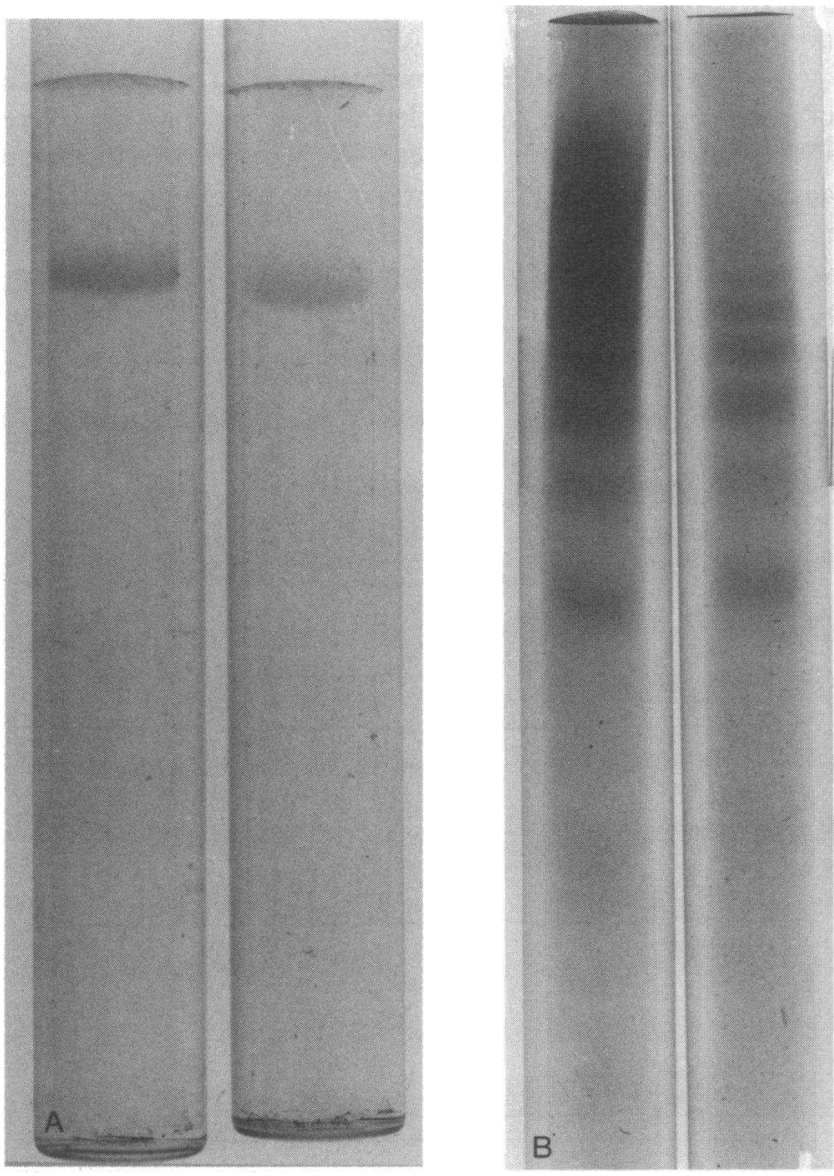

Figure 5. (A) Polyacrylamide gel electrophoresis of reduced vWf in the presence of SDS. Left, normal vWf after reduction; right, vWf purified from the patient with $\mathrm{vWd}$. The $M_{\mathrm{r}}$ of the two bands is almost identical. No other bands are seen other than the $v W f$ subunit. The gel is stained with Coomassie blue. $(B)$ The $0.5 \%$ agarose- $2.0 \%$ acrylamide gel. Left, normal vWf; right, patient's purified vWf. The patient's vWf lacks the largest multimer seen in the normal vWf; however, the intermediate and smaller bands have an identical migration and no bands are seen other than those present in the normal vWf. The gel is stained with Coomassie Blue. of $\sim 55 \%$ of that seen in normal vWf (normal, $121 \pm 12 \mathrm{nM} / \mathrm{mg}$, $n=7$, patient mean 55,48 , and $62 \mathrm{nM} / \mathrm{mg}$ ) on two different purified preparations; penultimate, terminal, and total galactose were deficient in the patient's purified vWf. Total galactose content of normal vWf was $180 \pm 15 \mathrm{nmol} / \mathrm{mg}(n=4)$ and the patient's vWf contained $116 \mathrm{nmol} / \mathrm{mg}$, a deficiency of $36 \%$. When normal intact $\mathrm{vWf}$ was treated with $\beta$-galactosidase, $34 \pm 8 \mathrm{nmol} /$ $\mathrm{mg}(n=4)$ were released. Identical studies with the patient's $\mathrm{vWf}$ released $16 \mathrm{nmol} / \mathrm{mg}$, a $53 \%$ deficiency of terminal $\beta$-galactosidase-released vWf. When $\beta$-galactosidase was incubated with normal asialo vWf, $150 \pm 13 \mathrm{nmol} / \mathrm{mg}(n=4)$ was released, while with $\mathrm{vWd}, \mathrm{vWf} 82 \mathrm{nmol} / \mathrm{mg}$ was released, a $46 \%$ deficiency. The penultimate galactose is determined by subtraction of the terminal galactose released (treatment of intact vWf) from the galactose released from asialo $\mathrm{vWf}$. The penultimate galactose content of normal vWf was $116 \pm 10 \mathrm{nmol} / \mathrm{mg}$ and that of the patient's vWf was $66 \mathrm{nmol} / \mathrm{mg}$, a $43 \%$ deficiency.

The monoclonal antibody directed against GPIb (6D1) inhibited the SPA at doses as low as $2 \mu \mathrm{g} / \mathrm{ml}$ (mean, $4.1 \mu \mathrm{g} / \mathrm{ml}$; range, $2.0-8.5 \mu \mathrm{g} / \mathrm{ml} ; n=9$ ). The monoclonal antibody against the GPIIb/IIIa complex (10E5) also inhibited the platelet aggregation; however, much larger amounts of this monoclonal antibody were required to completely inhibit SPA (minimum concentration, $6 \mu \mathrm{g} / \mathrm{ml}$; mean, $8.5 \mu \mathrm{g} / \mathrm{ml}$; range, $6-13 \mu \mathrm{g} / \mathrm{ml} ; n$ =6) (Fig. 6).

Inhibitors of platelet function that were studied all had some effect on the SPA. Dibutryl cyclic AMP and $\mathrm{PGI}_{2}$ both completely inhibited SPA at relatively low doses (Fig. 7). EDTA at a final concentration of $5 \mathrm{mM}$ also totally blocked the SPA. In contrast, EDTA at a final concentration of $1 \mathrm{mM}$ only partially inhibited SPA (Fig. 8), and only partial inhibition of the total aggregation was observed with apyrase and acetylsalicylic acid when they were studied over a wide range of concentrations (Fig. 7). Hiruidin at a final concentration of $40 \mathrm{U} / \mathrm{ml}$ had no effect on the SPA of the patients' PRP. Identical results were found when the same platelet function inhibitors were added to normal platelets resuspended in patient plasma; i.e., $5 \mathrm{mM}$ EDTA, db-cAMP, and $\mathbf{P G I}_{2}$ all totally inhibited aggregation, while EDTA $1 \mathrm{mM}$, acetylsalicylic acid, and apyrase only partially inhibited SPA.

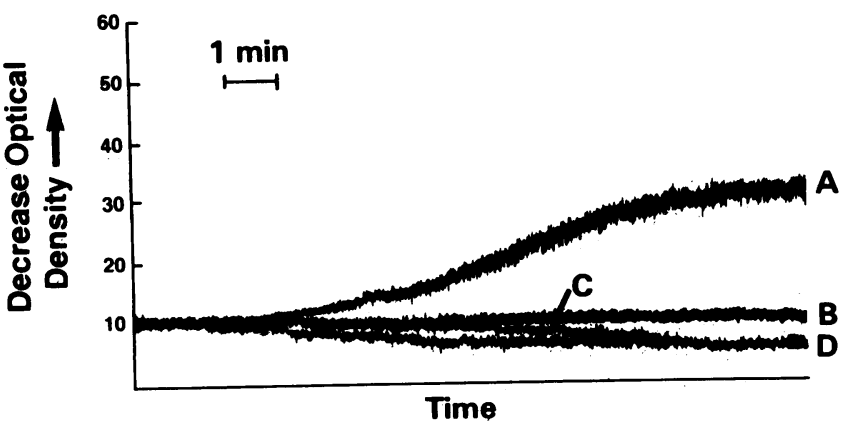

Figure 6. Platelet aggregation studies with monoclonal antibodies. PRP $0.4 \mathrm{ml}(200,000 / \mu \mathrm{l})$ was placed in the aggregometer at $37^{\circ} \mathrm{C}$ and stirred at $900 \mathrm{rpm}$. Tracing A is the SPA of patient PRP. Tracing B is the normal PRP showing no aggregation. Tracing $C$ is the patient PRP with the addition of the antibody against the GPIb (6D1). In nine experiments an average of $5 \mu \mathrm{g} / \mathrm{ml}$ of $6 \mathrm{D} 1$ totally inhibited the SPA. Tracing $D$ is the patient's PRP with the antibody directed against the GPIIb/IIla. In six experiments a mean of $8.5 \mu \mathrm{g} / \mathrm{ml}$ of $10 \mathrm{E} 5$ totally inhibited aggregation. 


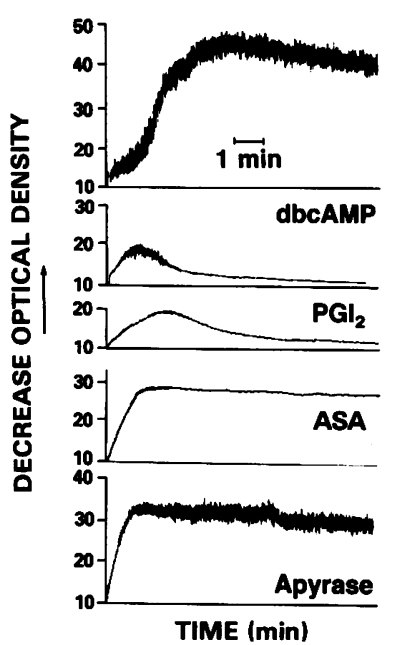

Figure 7. Inhibition of SPA by inhibitors of platelet function. At the top is patient PRP with only buffer added in place of an equal volume of the inhibitors. The next two panels, db-cAMP and $\mathrm{PGI}_{2}$, both show an initial wave of aggregation and then disaggregation. Both the db-cAMP and PGI $_{2}$ also inhibited ATP release. The minimum inhibitory concentration of db-cAMP was $1 \mathrm{mM}$ and of $\mathrm{PGI}_{2}$ was 10 $\mathrm{mM}$. The last two tracings show the effects of adding acetylsalicylic acid and apyrase to the PRP. Both show a reduction in the total extent of aggregation with virtually no change in the initial slope. Both acetylsalicylic acid and apyrase totally inhibited the ATP release. The minimal concentration of apyrase and acetylsalicylic acid that showed partial inhibition of the SPA, was $20 \mu \mathrm{g} / \mathrm{ml}$ and $0.23 \mathrm{mg} / \mathrm{ml}$, respectively. When the dose of apyrase and acetylsalicylic acid were increased to maximal doses (acetylsalicylic acid, $0.9 \mathrm{mg} / \mathrm{ml}$ and apyrase, $200 \mu \mathrm{g} / \mathrm{ml}$ ), no further inhibition was seen. Not shown is the tracing of PRP with hiruidin ( $20 \mathrm{U} / \mathrm{ml}$ final concentration) where there was no inhibition of the SPA.

\section{Discussion}

Variant forms of vWd with enhanced RIPA have been described which show similar characteristics to those of our four patients, including the increased aggregation of PRP to low-dose ristocetin, the altered multimeric structure of plasma vWf, and the occurrence of thrombocytopenia $(1-5,20-23)$. Type IIb vWd is described as a plasma abnormality, since the patients' plasma vWf binds to normal platelets at lower ristocetin concentrations than does normal $v W f(1)$. The platelets from these patients bind normal vWf in a normal manner. In contrast the platelets from individuals with pseudo- or platelet-type vWd aggregate only in the presence of added normal cryoprecipitate or plasma, suggesting that it is a primary platelet defect and providing a possible explanation for the absence of the largest plasma $v W f$ multimers and the thrombocytopenia in this disorder (2-4). Takahashi has also described a form of $\mathrm{vWd}$ with enhanced RIPA in which the patients' platelets bind more normal plasma vWf at low doses of ristocetin than do normal platelets (21-23). We have previ-

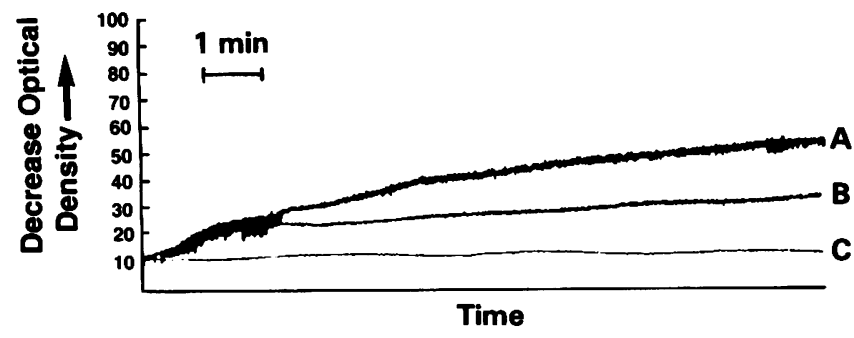

Figure 8. Aggregation of patient PRP and the effects of EDTA on SPA. Tracing A is the patient's PRP demonstrating SPA. Tracing B is sodium citrate at $1 \mathrm{mM}$ EDTA showing partial inhibition. Tracing $C$ is the same PRP which has been collected in an anticoagulant-containing sodium citrate and $5 \mathrm{mM}$ EDTA showing complete inhibition of the SPA. ously described a form of vWd similar to that described by Takahashi; however, in our binding studies we employed patients' platelets free of plasma proteins and used purified radiolabeled normal F.VIII/vWf (20). Takahashi et al. (23) have reported an abnormality of their patients' GPIb and have provided data to indicate that the patients have pseudo-vWd or platelet-type vWd. The patients presented here are different from those previously described in that their PRP invariably undergoes SPA, the patients' plasmas cause aggregation of normal platelets, the SPA is dependent on the integrity of both the GPIb and GPIIb/ IIIa, and plasma fibrinogen is an essential plasma cofactor for the SPA.

SPA has not been a common finding in vWd. Miller et al. (6) did not detect any SPA in their patients with platelet-type vWd. They observed platelet aggregation in the platelet-type vWd only after adding or infusing normal vWf in the form of plasma cryoprecipitate or purified vWf $(4,6)$. Weiss et al. (2) also stated that their patients with pseudo-vWd did not undergo SPA, and that their platelets aggregated only after a source of normal vWf was added to the patient's PRP. SPA has not been detected in type IIb vWd. Recently two families have been reported with SPA. They include one with platelet-type vWd who showed slight SPA which was markedly augmented by the addition of normal plasma (23), and one other study with SPA (24). In the latter study, two patients had defects of their platelets and two defects in their plasma when studied in perfusion experiments (24). Holmberg et al. (5) described SPA and the development of thrombocytopenia in a patient with type IIb $\mathrm{vWd}$ who was treated with DDAVP. They suggested that the mechanism for the post-DDAVP aggregation was related to the release of the largest vWf multimers from endothelial cells. The four patients reported here differ in several ways from those previously reported in that all four patients consistently have had SPA and that the addition of normal plasma cryoprecipitate or purified vWf did not augment the SPA. Our patients' platelets did not undergo SPA when resuspended in normal plasma containing normal vWf as was seen with the platelet-type vWd and pseudovWf. The native plasma of our patients was capable of inducing the SPA independent of DDAVP infusion or the addition of normal vWf.

We have characterized the protein(s) in our patients' plasma, which induces SPA. The purified vWf from one of our patients induced platelet aggregation in the PRP from normal subjects or a patient with severe $\mathrm{vWd}$. It also induced aggregation when normal or severe $v W d$ platelets were resuspended in the plasma from a patient with severe vWd. However, when normal platelets were resuspended in afibrinogenemic plasma, the patients' purified vWf did not induce aggregation. This indicates that fibrinogen is an important plasma cofactor for the expression of the SPA and that the presence of normal plasma vWf is not necessary. These studies also show that platelet $\mathrm{vWf}$ did not play a role in the SPA, i.e., the platelets or PRP from the patient with severe vWd (<3\% platelet vWf activity and VIII R:Ag), aggregated to the same extent as normal platelets when suspended in the patients' plasma or when the patient's purified vWf was added the PRP.

Recent studies in platelet-type vWd in Japanese patients have shown an abnormality of the GPIb, possibly explaining why the platelets of these patients adsorbed normal vWf without any agonist (23). The GPIlb and GPIII were reported to be normal. Studies of the platelet glycoproteins involved in the SPA indicate that both GPIb and the GPIIb/IIIa complex are necessary for 
SPA. Monoclonal antibodies against either the GPIb or GPIIb/ IIIa complex totally inhibit the SPA of the patients' PRP. The amount of monoclonal antibody directed against the GPIIb/IIIa complex that completely inhibited aggregation was approximately two times greater than the amount of the antibody directed against the GPIb.

Intrinsic platelet function is also important in SPA. The patients' SPA was associated with dense granule release and alpha granule release, and metabolically inactive (formalinized) platelets were not aggregated by the patients' plasma even when exogenous calcium and/or magnesium ions were added. SPA is dependent on intact platelet metabolism and calcium ions (see below), indicating that the mechanism of SPA is not an agglutination reaction.

EDTA inhibits the SPA at a concentration of $5 \mathrm{mM}$, and only partially at a concentration of $1 \mathrm{mM}$. Calcium ions may be important in several reactions necessary for SPA including the maintenance of a stable GPIIb/IIIa complex, for binding of plasma vWf or fibrinogen to platelet glycoprotein receptor(s) or for the aggregation process. EDTA added in vitro inhibits the platelet aggregation of the PRP of platelet-type $\mathrm{vWd}$ seen after adding cryoprecipitate or plasma and the post-DDAVP platelet aggregation in type IIb vWd patients $(2,5,23)$. Calcium ions are also necessary for the platelet aggregation that occurs after addition of AS-vWf to platelets (see below) (7-10, 25).

Several platelet metabolic pathways seem to play a role in SPA. PGI $_{2}$ has been shown to partially inhibit the ristocetininduced platelet aggregation or the bovine vWf-induced aggregation of normal PRP (26), and to totally inhibit the plasma or cryoprecipitate-induced aggregation of PRP from platelet-type or pseudo-vWd patient $(2,23)$. The mechanism of this $\mathrm{PGI}_{2}$ inhibition of platelet aggregation-induced by $\mathrm{vWf}$ in these patients is not entirely clear, however. The level of cAMP in particular may be important (Fig. 7). Fujimoto et al. (27) have shown that $\mathrm{PGI}_{2}$ and/or db-cAMP interfere with thrombin-induced vWf binding to human platelets and will also reverse the binding of radiolabeled vWf to the platelet receptor. Hawiger et al. (28) and Graber and Hawiger (29) reported that cyclic AMP levels regulate the exposure of the fibrinogen receptor in human platelets. These findings agree with recent evidence showing that $\mathrm{vWf}$ binding in the presence of thrombin or ADP occurs to the GPIIb/ IIIa complex at or near the same site as the fibrinogen receptor (30-35). The inhibition of the SPA in these patients by db-cAMP, $\mathrm{PGI}_{2}$, and by the monoclonal antibody $10 \mathrm{E} 5$ agrees with our findings that the GPIIb/IIIa complex plays an important role in the SPA. Inhibition of the exposure of the fibrinogen receptor by elevated cAMP levels or blocking the receptor by the monoclonal antibody directed against this glycoprotein both totally inhibit the SPA.

Apyrase and acetylsalicylic acid have been reported to have differing effects in platelet-type vWd and pseudo-vWd. Miller et al. (6) state that apyrase has no effect on the normal vWfinduced aggregation of the patient PRP, while Weiss et al. (2) and Takahashi et al. (23) have reported a partial inhibition of this aggregation by the presence of acetylsalicylic acid. Our studies indicate that both apyrase and acetylsalicylic acid primarily inhibit the total extent of aggregation rather than the initial slope of aggregation (Fig. 7). In our studies, apyrase at concentrations that totally inhibit ADP-induced platelet aggregation in normal PRP only partially inhibits the SPA (total aggregation decreased by $34 \%$ ), indicating that the aggregation was not totally related to the release of ADP. Acetylsalicylic acid inhibits $42 \%$ of total aggregation, indicating that the thromboxane synthetic pathway also plays only a partial role in the SPA.

Purified vWf from one of these four patients contained only $45 \%$ of the sialic acid and $54 \%$ of the penultimate galactose concentration of normal vWf. Asialo vWf (AS vWf) has been shown to aggregate normal platelets in the absence of ristocetin $(7-10,25)$. We and others have recently shown that AS-vWf binds to both the GPIb and the GPIIb/IIIa complexes $(10,11)$. The initial step is binding to GPIb followed by binding to GPIIb/ IIla and subsequent aggregation. Others have described $(7,25)$, and we have confirmed, that EDTA markedly inhibits the ASvWf aggregation process (11; and Gralnick, H. R., S. B. Williams, and L. McKeown, unpublished observation). Plasma fibrinogen is an essential cofactor for the induction of AS-vWf platelet aggregation, and db-cAMP, $\mathrm{PGI}_{2}$, and EDTA totally inhibit the AS-vWf-induced platelet aggregation and acetylsalicylic acid and apyrase only partially inhibit the AS-vWf-induced platelet aggregation (35). We have recently found that the AS-agalacto $v W f$ (removal of the terminal and penultimate galactose) binds to and causes platelet aggregation in normal PRP. The aggregation induced by the AS-agalacto vWf, like the AS-vWf, is dependent on the presence of plasma fibrinogen (36).

The in vitro findings with AS-vWf are identical to those currently reported in our four patients with vWd. Circulating AS$v W f$ or AS-agalacto vWf would explain the results seen in our patients: (a) The presence of AS-vWf in the patients' plasma would result in spontaneous aggregation of their platelets and normal platelets. (b) Thrombocytopenia would result from in vivo platelet aggregation and removal of aggregates from the circulation. (c) AS-vWf in the patient's PRP would result in enhanced RIPA. The abnormalities found in these patients are different from those previously described in other $\mathrm{vWd}$ variants that: $(a)$ the platelet aggregation is not ristocetin-dependent, $(b)$ both GPIb and GPIIb/IIla are required for platelet aggregation, (c) an exogenous source of normal $\mathrm{vWf}$ is not necessary for platelet aggregation, and $(d)$ fibrinogen is essential for the SPA. Although this form of $\mathrm{vWd}$ is different from those previously reported, it may have one or more pathophysiologic mechanisms in common with either pseudo-vWd or platelet-type vWd, or type Ilb vWd. The SPA seen in these patients with $v W d$ is a complex reaction that involves both the GPIb and GPIIb/IIIa receptors, plasma fibrinogen, the presence of free calcium ions, and metabolically active platelets.

\section{References}

1. Ruggeri, Z. M., F. I. Pareti, P. M. Mannucci, N. Ciavarella, and T. S. Zimmerman. 1980. Heightened interaction between platelets and factor VIII/von Willebrand factor in a new subtype of von Willebrand's disease. N. Engl. J. Med. 302:1047-1051.

2. Weiss, H. J., D. Meyer, R. Rabinowitz, G. Pietu, J.-P. Girma, W. J. Vicic, and J. Rogers. 1982. Pseudo-von Willebrand's disease. An intrinsic platelet defect with aggregation by unmodified human factor VIII/von Willebrand factor and enhanced adsorption of its high-molecular weight multimers. N. Engl. J. Med. 306:326-333.

3. Miller, J. L., and A. Castella. 1982. Platelet-type von Willebrand's disease: characterization of a new bleeding disorder. Blood. 60:790-794.

4. Miller, J. L., B. D. Boselli, and J. M. Kupinski. 1984. In vivo interaction of von Willebrand factor with platelets following cryoprecipitate transfusion in platelet-type von Willebrand's disease. Blood. 63: 226-230.

5. Holmberg, L., I. M. Nilsson, L. Borge, M. Gunnarsson, and E. Sjorin. 1983. Platelet aggregation induced by I-desamino-8-D-arginine 
vasopressin (DDAVP) in type IIb von Willebrand's disease. $N$. Engl. J. Med. 309:816-821.

6. Miller, J. L., J. M. Kupinski, A. Castella, and Z. M. Ruggeri. 1983. von Willebrand factor binds to platelets and induces aggregation in platelet-type but not type IIb von Willebrand disease. J. Clin. Invest. 72 : 1532-1542.

7. Vermylen, J., M. B. Donati, G. de Gaetano, and M. Verstraete. 1973. Aggregation of human platelets by bovine or human factor VIII: role of carbohydrate side chains. Nature (Lond.). 244:167-168.

8. Vermylen, J., G. de Gaetano, M. B. Donati, and M. Verstraete. 1974. Platelet-aggregating activity in neuraminidase-treated human cryoprecipitates: its correlation with factor VIII-related antigen. $\mathrm{Br}$. J. Haematol. 26:645-650.

9. Vermylen, J., D. Bottecchia, and H. Szpilman. 1976. Factor VIII and human platelet aggregation. Further studies on aggregation of human platelets by neuraminidase-treated human factor VIII. Br. J. Haematol. 34:321-330.

10. Gralnick, H. R., S. B. Williams, and B. S. Coller. 1985. Asialo von Willebrand factor interactions with platelets. Interdependence of glycoproteins Ib and IIb/IIla for binding and aggregation. J. Clin. Invest. 75:19-25.

11. DeMarco, L., A. Girolami, S. Russell, and Z. M. Ruggeri. 1985. Interaction of asialo von Willebrand factor with glycoprotein Ib induces fibrinogen binding to the glycoprotein IIb/IIla complex and mediates platelet aggregation. J. Clin. Invest. 75:1198-1203.

12. Gralnick, H. R., B. S. Coller, and Y. Sultan. 1975. Studies of the human factor VIII/von Willebrand factor protein. III. Qualitative defects in von Willebrand's disease. J. Clin. Invest. 56:814-820.

13. Gralnick, H. R., S. B. Williams, and D. K. Morisato. 1981. Effect of the multimeric structure of the factor VIII/von Willebrand factor protein on binding to platelets. Blood. 58:387-392.

14. Corash, L., B. Shafer, and M. Perlow. 1978. Heterogeneity of human whole blood platelet subpopulations. II. Use of a subhuman primate model to analyze the relationship between density and platelet age. Blood. 52:726-732.

15. Coller, B. S., E. I. Peerschke, L. E. Scudder, and C. Sullivan. 1983. Studies with a murine monoclonal antibody that abolishes ristocetin-induced binding of von Willebrand factor to platelets: additional evidence in support of GPIb as a platelet receptor for von Willebrand factor. Blood. 61:99-110.

16. Coller, B. S., E. I. Peerschke, L. E. Scudder, and C. A. Sullivan. 1983. A murine monoclonal antibody that completely blocks the binding of fibrinogen to platelets produces a thrombasthenic-like state in normal platelets and binds to glycoproteins IIb and/or IIIa. J. Clin. Invest. 72: 325-338.

17. Warren, L. 1959. The thiobarbituric acid assay of sialic acids. $J$. Biol. Chem. 234:1921-1975.

18. Finch, R. P., R. Yuen, H. Schacter, and M. A. Moscarello. 1969. Enzymatic methods for the micro assay of D-mannose, D-glucose, Dgalactose and L-fucose from acid hydrolyzates of glycoproteins. Anal. Biochem. 31:296-301.

19. Gralnick, H. R., M. C. Cregger, and S. B. Williams. 1982. Characterization of the defect of the factor VIII/von Willebrand factor protein in von Willebrand's disease. Blood. 59:542-548.
20. Hoyer, L. W., and J. R. Shainoff. 1982. Factor VIII-related protein circulates in normal human plasma as high molecular weight multimers. Blood. 60:790-794.

21. Takahashi, H. 1980. Studies on the pathophysiology and treatment of von Willebrand's disease. IV. Mechanism of increased ristocetin-induced platelet aggregation in von Willebrand's disease. Thromb. Res. 19:857-867.

22. Takahashi, H., N. Sakuragawa, and A. Shibata. 1980. von Willebrand's disease with an increased ristocetin-induced platelet aggregation and a qualitative abnormality of the factor VIII protein. Am. J. Hematol. 8:299-308.

23. Takahashi, H., M. Handa, K. Watanabe, Y. Ando, R. Nagayama, A. Hattori, A. Shibata, A. B. Federici, Z. M. Ruggeri, and T. S. Zimmerman. 1984. Further characterization of platelet-type von Willebrand's disease in Japan. Blood. 64:1254-1262.

24. Sakariassen, K. S., H. K. Nieuwenhuis, M. Ottenhof-Rovers, and J. J. Sixma. 1985. Differentiation of patients with subtype IIb-like von Willebrand's disease by means of perfusion experiments with reconstituted blood. Br. J. Haematol. 59:459-470.

25. De Marco, L., and S. S. Shapiro. 1981. Properties of human asialo-factor VIII. A ristocetin-independent platelet-aggregating agent. J. Clin. Invest. 68:321-328.

26. Coller, B. S. 1981. Inhibition of von Willebrand factor-dependent platelet function by increased platelet cyclic AMP and its prevention of cytoskeleton-disrupting agents. Blood. 57:846-855.

27. Fujimoto, T., S. Ohara, and J. Hawiger. 1982. Thrombin-induced exposure and prostacyclin inhibition of the receptor of factor VIII/von Willebrand factor on human platelets. J. Clin. Invest. 69:1212-1222.

28. Hawiger, J., S. Parkinson, and S. Timmons. 1980. Prostacyclin inhibits mobilization of fibrinogen-binding sites on human ADP- and thrombin-treated platelets. Nature (Lond.). 283:195-197.

29. Graber, S. E., and J. Hawiger. 1982. Evidence that changes in platelet cyclic AMP levels regulate the fibrinogen receptor on human platelets. J. Biol. Chem. 257:14606-14609.

30. Ruggeri, Z. M., R. Bader, and L. de Marco. 1982. Glanzmann's thrombasthenia: deficient binding of von Willebrand factor to thrombinstimulated platelets. Proc. Natl. Acad. Sci. USA. 79:6038-6041.

31. Ruggeri, Z. M., L. de Marco, L. Gatti, R. Bader, and R. R. Montgomery. 1983. Platelets have more than one binding site for von Willebrand factor. J. Clin. Invest. 72:1-12.

32. Schullek, J., J. Jordan, and R. R. Montgomery. 1984. Interaction of von Willebrand factor with human platelets in the plasma milieu. $J$. Clin. Invest. 73:421-428.

33. Gralnick, H. R., S. B. Williams, and B. S. Coller. 1984. Fibrinogen competes with von Willebrand factor for binding to the glycoprotein IIb/IIla complex when platelets are stimulated with thrombin. Blood. 64:797-800.

34. Plow, E. F., A. H. Srouji, D. Meyer, G. Marguerie, and M. H. Ginsburg. 1984. Evidence that three adhesive proteins interact with a common recognition site on activated platelets. J. Biol. Chem. 259:53885391.

35. Pietu, G., G. Cherel, G. Marguerie, and D. Meyer. 1984. Inhibition of von Willebrand factor-platelet interaction by fibrinogen. Nature (Lond.). 308:648-649. 